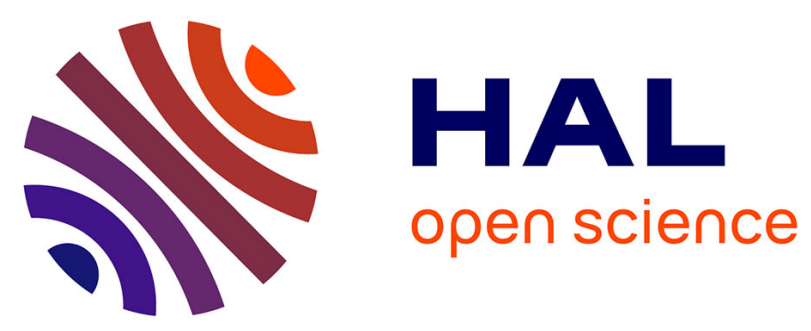

\title{
Fuzzy interpretation of performance scorecards for decision-making in the industrial context
}

Laurent Foulloy, Vincent Clivillé, Lamia Berrah

\section{To cite this version:}

Laurent Foulloy, Vincent Clivillé, Lamia Berrah. Fuzzy interpretation of performance scorecards for decision-making in the industrial context. 19th World Congress of the International Federation of Automatic Control | Promoting automatic control for the benefit of humankind, Aug 2014, Cape Town, South Africa. hal-01095676

\section{HAL Id: hal-01095676 https://hal.science/hal-01095676}

Submitted on 16 Dec 2014

HAL is a multi-disciplinary open access archive for the deposit and dissemination of scientific research documents, whether they are published or not. The documents may come from teaching and research institutions in France or abroad, or from public or private research centers.
L'archive ouverte pluridisciplinaire HAL, est destinée au dépôt et à la diffusion de documents scientifiques de niveau recherche, publiés ou non, émanant des établissements d'enseignement et de recherche français ou étrangers, des laboratoires publics ou privés. 


\title{
Fuzzy interpretation of performance scorecards for decision-making in the industrial context
}

\author{
Laurent Foulloy*, Vincent Clivillé*, Lamia Berrah* \\ *LISTIC Polytech Annecy Chambéry, France, \{e-mail: laurent.foulloy@univ-savoie.fr, vincent.cliville@univ-savoie.fr, \\ lamia.berrah@univ-savoie.fr)\}
}

\begin{abstract}
This article deals with the use, for industrial decision-making, of performance expressions that are provided by performance indicators and performance measurement systems. Two kinds of expression are distinguished in this sense: the performance measure on the one hand, and the performance evaluation on the other hand. While the performance measure identifies the achievement degree of the assigned objective, the performance evaluation handles the judgement of the decision-maker with regards to the obtained result. We focus in this study on the definition of a particular performance evaluation mechanism, which consists on a fuzzy symbolic handling of performance measures. Structural objective break-down that allows decision-makers to define the links between strategic, tactical and operational levels is the considered framework. We assume that performance measures are expressed at the operational level, for local control purposes, and look for translating them into performance evaluations, making them useful for the tactical decision-making. Performance measures are defined under numerical values. Fuzzification and defuzzification approaches are used in order to describe these values in linguistic terms, such as bad, medium or good. Symbolic items are also proposed. Some illustrations are presented, extracted from a case study which has been carried out in the Bosch Rexroth S.A. Company.
\end{abstract}

\author{
Keywords: Decision-making - Industrial objectives - Performance expression - Structural \\ breakdown - Fuzzy symbolic handling.
}

\section{INTRODUCTION}

This study deals with the wide problem of providing useful pieces of information for decision-making in the industrial improvement context. Scorecards are generally defined in this sense [Kaplan and Norton (1992)], [Epstein and Mansoni (1998)], [Bourguignon et al. (2004)], and deal with the different criteria that are involved in the decision-making process. Scorecards are constituted from a set of performance expressions that reflect the achievement of the assigned objectives. Decisions are taken and action plans are launched in order to either achieve the objectives better or declare others that are more adequate with the considered system capacities.

In the industrial context, decisions are generally taken at the strategic and tactical levels, involving a set of diversified criteria. Such decisions are made by decision-makers who are often at the head of the considered business units and services.

Our purpose in this work subscribes to a relevant use, by the decision-makers, of the expressed performances in order to make their decisions. We describe here one manner that the decision-makers can use for interpreting the obtained performance expressions, before acting.
To be more precise, our idea consists in considering two kinds of performance expressions: the performance measure and the performance evaluation. While a performance measure is defined as the direct comparison of the reached measures and the declared objectives, a performance evaluation handles a judgement semantic, in terms of satisfaction or happiness of the decision-maker, with regards to the obtained results. Such an expression consists generally in the translation of numerical value into a qualitative linguistic one, or other symbols that can transmit the judgement of the decision-makers (colours, smileys...).

Besides, beyond its declaration aspect, an objective can be considered from its achievement point of view, namely the evolution of the associated action plan. Indeed, one can summarise the procedure as follows. First, an objective is declared, namely through a target value which is associated with a variable or criterion and which should be achieved at the end of a given temporal horizon [Kaplan and Norton (1996)], [Vallespir et al. (1993)], [Clivillé et al. (2007)], [Gomez et al. (2001)]. An action plan is simultaneously defined in order to achieve the objective. If the objective is "simple", then the action is immediate. But if the objective is "complex", overall or strategic, the action plan is deployed according to "structural" break-downs, on the one hand and "temporal" ones on the other hand. The former concerns the 
representation of trees of variables, the variables being related between them by coordination and contribution links. Structured hierarchical representations are used in order to highlight the contribution link between the three decisional levels, namely the strategic, the tactical and the operational ones. The operational level identifies the operational actions that are locally controlled, while the tactical level identifies the intermediate level between the strategy and the operational actions. At this level, decisions are taken with regards to the obtained operational results on the one hand and to the strategy on the other hand. Scorecards or aggregated performances can thus be used at this level for the decision-making.

The aim of this study deals with the definition of fuzzy mechanisms in order to turn performance measures into performance evaluations, by considering a performance quantification framework previously developed by our team. In Section 2, we recall the major principles of the performance expression, as studied in our previous works. In order to illustrate our ideas, the decision problem which has been submitted by the Bosch Rexroth S.A. Company is presented in Section 3. Section 4 is thus dedicated to the proposed formalism. Finally, some remarks and perspectives conclude this study.

\section{BACKGROUND ON PERFORMANCE EXPRESSION}

Over the last fifteen years, the literature, in accordance with the industrial decision-makers' point of view, has provided many studies and analyses concerning the performance concept [Lebas (1995)], its expression [Berrah et al. (2000)], the performance indicators (PI's) [Fortuin (1988)] and the performance measurement systems (PMS's) as tools for its measurement [Nudurupati et al. (2011)], [Neely et al. (1995)], [Nair (2006)]. More particularly, we proposed in previous works [Berrah et al. (1998)] to define the performance expression by a function $P$, such that $p=P(o, m)$ where $O, M$ and $E$ are respectively the universes of discourse of the set of objectives $o$, the set of measures $m$ and the set of the performance expressions $p$.

Moreover, we focused on making the difference between two different kinds of performance expression, the "performance measure" on the one hand and the "performance evaluation" on the other hand [Berrah et al. (2004)]. Formally, the performance measure identifies the comparison of the measure to the target value, without any condition on $E$. Such an expression is often used for tactical and operational decisions, generally for well-located business units. While the performance measure handles the achievement degree of the objective, the performance evaluation transmits beyond this piece of information, the judgement or the satisfaction of the decision-maker with regard to the obtained score [Belton and Stewart (2002)]. The performance evaluation constitutes thus an analysis element for aiding the decision-maker to react and decide what to do in the given context. Such an expression can be then considered as a first interpretation of the performance measure, which is more absolute and objective. Formally, it was chosen to define the performance evaluation on $[0,1]$ universe or other qualitative universes such as $\{\mathrm{bad}$, medium, good $\}$.

For the sake of illustration, the case study is presented first in order to present our proposals of translating performance measures into performance evaluations.

\section{CASE STUDY}

\subsection{Presentation}

The Bosch Rexroth S.A. Company is a world leader in the industrial automation components market. More particularly, the business unit in France designs and produces cylinders and distributors for automation with about 400 employees and a turnover of about 60 million $€$. The company manufactures standardized items including 28,500 hydraulic cylinders. The variety of products is very wide especially concerning the hydraulic cylinders (a few million possibilities). The manufacturing process is weakly automated, consisting of activities of manufacturing, assembly, finishing, packaging and dispatching. The product is made of about 15 to 25 elementary parts. The manufacturing cycle time is between 2 and 10 days according to the process described in Fig. 2.

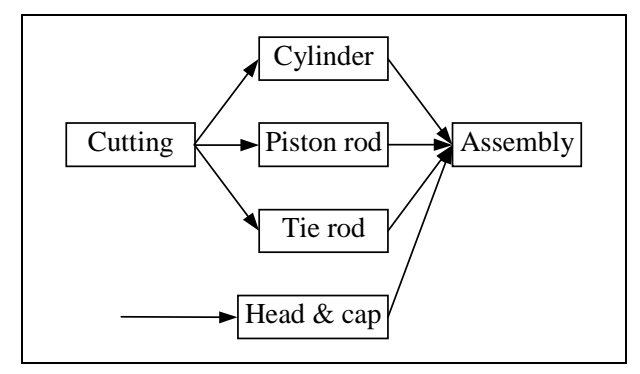

Fig. 2: The activities of the Hydraulic Cylinder (HC) Process

Since the beginning of the 80's, the Bosch Rexroth S.A. Company has adopted and progressively generalized continuous improvement approaches, according to the Toyota Production System. These ones concern the classical performance criteria such as quality, productivity, safety, customer satisfaction and environment and also include the logistics, management and lean manufacturing aspects. The submitted case focuses on the On Time Delivery (ODT) tactical objective. The ODT is considered as being important for manufacturing domain and is related to the $\mathrm{HC}$ process. At the beginning of 2012, its $46 \%$ level $46 \%$ was not satisfying the plant manager. Hence, an improvement action plan has been developed and progressively executed, along a temporal horizon that goes from the beginning of 2012 to the end of 2013. To define and control this action plan, the objective, which has been declared at the tactical level, has been broken-down according to a both structural and temporal approach. The structural break-down highlights the operational objectives, while the temporal break-down gives the achievement of objective temporal trajectory.

Concerning the structural break-down, the objective tree is the result of a quantification procedure of a preliminary 
variable tree (Fig. 3). In our case, the variables are deduced from the activities described in Fig. 1. Each branch is identified to each activity. For the Assembly branch, three leaves (variables) are considered. Note that for the sake of conciseness, only the final assembly line is displayed, but measures and analyses concerning the cylinder, the tie rod, etc. have been also studied.

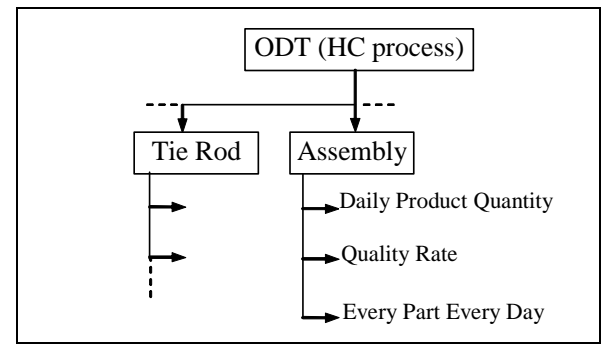

Fig. 3: The variable tree related to the ODT with regards to the HC process.

For each variable an objective is defined à $t_{f}$, i.e. the end of the temporal horizon, namely the end of the action plan:

- the productivity, defined by the ratio of the items number produced every day compared to the maximum capacity of 120 items instanced in the Daily Production Quantity (DPQ), $o\left(D P Q, t_{f}\right)=100 \%$,

- the Quality Rate, measured by the ratio of the product unconformities $(\mathrm{QR}), o\left(Q R, t_{f}\right)=2 \%$,

- the agility, identified to the number of needed days to produce the whole types of items and instanced in the Every Part Every Day (EPED), $o\left(E P E D, t_{f}\right)=2$ days .

For each tactical or operational objective, a trajectory is defined in order to give reference information for the plant manager during the action plan. More precisely, intermediate values are defined for each monthly meeting. The OTD trajectory is defined in a linear way, from the initial value of $46 \%$ to the final expected one of $80 \%$ (Fig. 4).

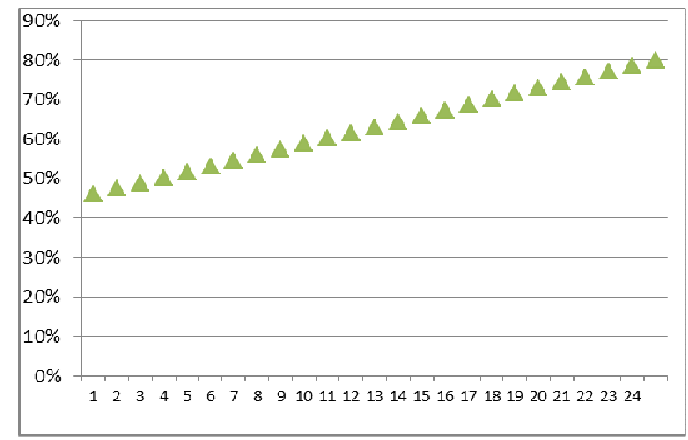

Fig. 4: The ODT objective trajectory.
Let $t_{i}$ be the milestone for the $i^{t h}$ month. Hence, the intermediate objective at $t_{i}$ is obtained thanks to the following relation:

$$
o\left(t_{i}\right)=o\left(t_{0}\right)+i . \frac{\left(o\left(t_{24}\right)-o\left(t_{0}\right)\right)}{24}, i=1 \text { to } 24 .
$$

In the same way the operational objectives are defined along the improvement duration by plateaus from 2 to 12 months (table 1). For sake of conciseness, only the data of the first 9 months of the improvement action plan are given.

To control his action plan the plant manager needs to be informed each month about the OTD objective achievement. Tactical performance expressions and also operational performance ones are useful in this sense.

\subsection{Needs of decision-making}

Data are available to know the reaching degree of the brokendown objectives in Table 1. But from the plant manager's point of view, data are not totally meaningful to control the action plan. Indeed, the plant manager has to take into account more than twenty operational performance measures according to the whole activities of the $\mathrm{HC}$ process. Obviously he is able to give sense to the OTD measure knowing the objective, but it is more difficult for the operational measures. So it is proposed to express the performance by comparing the measure to the objective.

Table 1: Operational objectives and measures for the first 9 months

\begin{tabular}{|l|c|c|c|c|c|c|c|c|c|c|}
\hline \multicolumn{10}{|c|}{ Hydraulic cylinder On Time Delivery } \\
\hline ti in month & 0 & 1 & 2 & 3 & 4 & 5 & 6 & 7 & 8 & 9 \\
\hline $\mathrm{o}(\mathrm{OTD}, \mathrm{ti})$ & $46 \%$ & $47,4 \%$ & $48,8 \%$ & $50,3 \%$ & $51,7 \%$ & $53,1 \%$ & $54,5 \%$ & $55,9 \%$ & $57,3 \%$ & $58,8 \%$ \\
\hline $\mathrm{m}(\mathrm{OTD}, \mathrm{ti})$ & $46,0 \%$ & $46,0 \%$ & $46,6 \%$ & $47,5 \%$ & $48,2 \%$ & $50,0 \%$ & $52,5 \%$ & $51,0 \%$ & $51,1 \%$ & $52,9 \%$ \\
\hline \multicolumn{8}{|c|}{ inal assembly line } \\
\hline $\mathrm{o}(\mathrm{DPQ}, \mathrm{ti})$ & & $66,7 \%$ & $66,7 \%$ & $70,8 \%$ & $70,8 \%$ & $75,0 \%$ & $75,0 \%$ & $79,2 \%$ & $79,2 \%$ & $79,2 \%$ \\
\hline $\mathrm{m}(\mathrm{DPQ}, \mathrm{ti})$ & $60,0 \%$ & $62,5 \%$ & $64,2 \%$ & $67,1 \%$ & $67,5 \%$ & $70,0 \%$ & $71,7 \%$ & $69,2 \%$ & $68,3 \%$ & $70,8 \%$ \\
\hline $\mathrm{o}(\mathrm{QR}, \mathrm{ti})$ & & $3,0 \%$ & $3,0 \%$ & $3,0 \%$ & $3,0 \%$ & $3,0 \%$ & $3,0 \%$ & $2,7 \%$ & $2,7 \%$ & $2,7 \%$ \\
\hline $\mathrm{m}(\mathrm{QR}, \mathrm{ti})$ & $3,4 \%$ & $3,2 \%$ & $3,1 \%$ & $2,9 \%$ & $3,0 \%$ & $2,9 \%$ & $2,8 \%$ & $2,9 \%$ & $2,8 \%$ & $2,6 \%$ \\
\hline $\mathrm{o}(\mathrm{EPED}, \mathrm{ti})$ in days & & 3 & 3 & 3 & 3 & 3 & 3 & 3 & 3 & 3 \\
\hline $\mathrm{m}(\mathrm{EPED}, \mathrm{ti})$ & 4,3 & 4,3 & 4,3 & 4,1 & 3,9 & 3,9 & 3,9 & 3,7 & 3,4 & 3,4 \\
\hline $\mathrm{p}(\mathrm{DPQ}, \mathrm{ti})$ & & $-4,2 \%$ & $-2,5 \%$ & $-3,7 \%$ & $-3,3 \%$ & $-5,0 \%$ & $-3,3 \%$ & $-10,0 \%$ & $-10,9 \%$ & $-8,4 \%$ \\
\hline $\mathrm{p}(\mathrm{QR}, \mathrm{ti})$ & & $-0,2 \%$ & $-0,1 \%$ & $0,1 \%$ & $0,0 \%$ & $0,1 \%$ & $0,2 \%$ & $-0,2 \%$ & $-0,1 \%$ & $0,1 \%$ \\
\hline $\mathrm{p}(\mathrm{EPED}, \mathrm{ti})$ & & $-1,3$ & $-1,3$ & $-1,1$ & $-0,9$ & $-0,9$ & $-0,9$ & $-0,7$ & $-0,4$ & $-0,4$ \\
\hline
\end{tabular}

After discussions, it was decided to adopt the difference operator $p\left(t_{i}\right)=o\left(t_{i}\right)-m\left(t_{i}\right)$ rather than the ratio $p\left(t_{i}\right)=m\left(t_{i}\right) / o\left(t_{i}\right)$, in order to avoid possible ambiguity with OTD, DPQ and QR which are expressed as ratios. Corresponding results are given in Table 2.

Table 2: values of operational performance expression

\begin{tabular}{|l|c|c|c|c|c|c|c|c|c|c|}
\hline $\mathrm{ti}$ in month & 0 & 1 & 2 & 3 & 4 & 5 & 6 & 7 & 8 & 9 \\
\hline $\mathrm{p}(\mathrm{DPQ}, \mathrm{ti})$ in \% & & $-4,2$ & $-2,5$ & $-3,7$ & $-3,3$ & -5 & $-3,3$ & -10 & $-10,9$ & $-8,4$ \\
\hline $\mathrm{p}(\mathrm{QR}, \mathrm{ti})$ in \% & & $-0,2$ & $-0,1$ & 0,1 & 0 & 0,1 & 0,2 & $-0,2$ & $-0,1$ & 0,1 \\
\hline $\mathrm{p}(\mathrm{EPED}, \mathrm{t})$ in days & & $-1,3$ & $-1,3$ & $-1,1$ & $-0,9$ & $-0,9$ & $-0,9$ & $-0,7$ & $-0,4$ & $-0,4$ \\
\hline
\end{tabular}

However, these performance expressions are not very helpful for the plant manager to take a decision. Indeed, these performance expressions are not commensurable and it is difficult to give a common global sense at this set of pieces of 
information. Significant pieces of information about each objective achievement should be interesting to build an opinion on the relevance of the launched actions. In other words, the plant manager has been happy with the idea to have the whole set of performance expressions being represented as simple as possible.

In this context, an evaluation on a linguistic scale, e.g. $L=\{$ Good, Medium, Bad $\}$, which qualifies the performance more intuitively is firstly proposed. Indeed, for instance, when the DPQ performance is Good the action plan is kept, when it is $\mathrm{Bad}$, it has to be corrected before the next measure, when it is Medium the gap must be explained. More complex scenarios can be taken into account. For example, two consecutive Bad DPQ can be necessary before correcting the action plan because it can be accepted that an exceptional event, corresponding to one unique measure, may disturb the company for a short period. It could also be envisioned that after three consecutive Medium DQP it is mandatory to correct the action plan. In order to put into place this mechanism, the plant manager has thus to give information in order to define what are the Good, Medium and Bad linguistic levels of performance.

\section{FROM PERFORMANCE MEASURE PERFORMANCE EVALUATION}

\subsection{Linguistic evaluation of the performance measure}

Associating linguistic information with numerical ones can be addressed by means of the concepts of fuzzy meaning proposed by Zadeh. Fuzzy meaning makes it possible to define a language as a fuzzy relation between the set of linguistic terms, $L=\{$ Good, Medium, Bad $\}$ here, and a universe of discourse $X$. The transformation between the set of linguistic terms and the related set of numbers can be formally defined by means of functions called the fuzzy meaning and the fuzzy description (also called descriptor set by Zadeh) [Zadeh (1971)].

Let $L$ be a set of linguistic terms and $X$ a set of numbers. Let $R$ be a fuzzy relation, i.e. a fuzzy subset of the Cartesian product $L \times X$, characterised by its membership function $\mu_{R}$. Let $Z$ be a set, $F(Z)$ denotes the set of all fuzzy subsets of $Z$. The fuzzy meaning of a term $l$ is given by the function $M: L \rightarrow F(X)$ defined by:

$\forall l \in L, \forall x \in X, \mu_{M(l)}(x)=\mu_{R}(l, x)$.

In the same manner, the fuzzy description of the number $x$ is given by the function $D: X \rightarrow F(L)$ defined by:

$\forall l \in L, \forall x \in X, \mu_{D(x)}(l)=\mu_{R}(l, x)$.

Because the fuzzy description transforms a number into a linguistic fuzzy set, i.e. a fuzzy set defined by a set of terms, it can be interpreted as a symbolic fuzzification, denoted $\varphi_{2}$ in previous work [Foulloy and Galichet (1995)], thus: $\varphi_{2}(x)=D(x)$.
Since the fuzzy meaning and the fuzzy description are two ways of characterising the relation $R$, we have the following equality:

$\forall l \in L, \forall x \in X, \mu_{D(x)}(l)=\mu_{M(l)}(x)$.

Hence, in the Bosch Rexroth S.A. Company case study, the set of fuzzy meanings represented in Fig. 4 are such that:

$\forall x \in X, \sum_{l \in L} \mu_{M(l)}(x)=1$ where $L=\{$ Good, Medium, Bad $\}$

In other words, the set of fuzzy meaning is a fuzzy partition according to Ruspini [Ruspini (1969)].

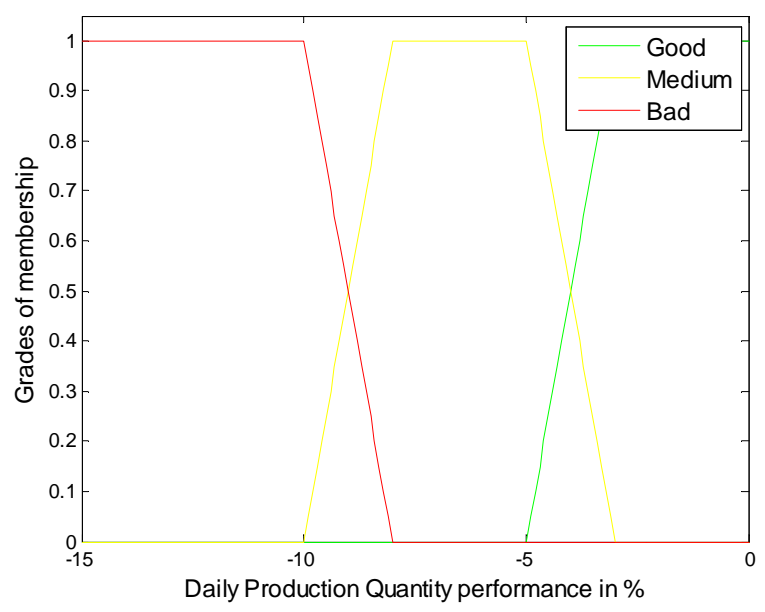

Fig. 4: Fuzzy partition of the DPQ performance.

At a given time $t$, thanks to the link between the fuzzy meaning and the fuzzy description, the fuzzy description of the performance measure $p(t)$ is such that:

$\sum_{l \in L} \mu_{D(p(t))}(l)=1$

According to Table 1, the DPQ objective $o\left(D P Q, t_{3}\right)=70.8 \%$ while the measure was $m\left(D P Q, t_{3}\right)=67.1 \%$ items. The performance measure, expressed by the difference between the objective and the measured rates is $p\left(D P Q, t_{3}\right)=-3.7 \%$. Using Zadeh's representation of fuzzy set [Zadeh (1965)], the performance evaluation is given by the fuzzy description of this value:

$D(-3.7)=0.65 /$ Good $+0.35 /$ Medium $+0 /$ Bad .

It means that the number -3.7 is respectively described by the term Good with a grade of membership equal to 0.65 , by the term Medium with a grade of membership equal to 0.35 and is not described at all by the term Bad since the grade of membership is equal to 0 .

\subsection{From linguistic evaluation to a user-friendly interface}


If the linguistic evaluation helps the decision-maker in his task, it is often preferred to have a more user-friendly interface like colours or smileys. For example, the color Red is often associated with a bad performance evaluation, and the happy smiley to a good performance evaluation.

Formally, this problem can be understood as transforming the linguistic fuzzy set, obtained by the symbolic fuzzification of the performance measure, to a new value in the space related to the user interface. This new value is, for example, a RGB vector for an interface with colours or the intensity of the smile for smileys. In both cases, this transformation can be interpreted as a symbolic defuzzification, denoted $\delta_{2}$ (see [Foulloy and Galichet (1995)]). Therefore, the performance measure transformation into user-friendly information can be represented by the composition of a symbolic fuzzification and a symbolic defuzzification:

$\forall x \in X, y=\delta_{2}\left(\varphi_{2}(x)\right) \in Y$

Generally, the symbolic defuzzification is a discrete case of the centre of gravity (COG) method. Let $z$ be a fuzzy set of $L$ and $\alpha_{i}$ be the grade of membership associated with each term $l_{i}$ in $L$. Assume also that $y_{i} \in Y$ are discrete values respectively associated with each term $l_{i}$, then we have:

$y=\delta_{2}(z)=\frac{\sum_{i} \alpha_{i} \cdot y_{i}}{\sum_{i} \alpha_{i}}$.

The previous equation is reduced to $y=\delta_{2}(z)=\sum_{i} \alpha_{i} \cdot y_{i}$ when fuzzy partitions are used since we have $\sum_{i} \alpha_{i}=1$.

\subsection{Colour-based user interface}

Let $L=\{$ Good, Medium, Bad $\}$. Let $Y=[0,1]^{3}$ be the set of normalised RGB (Red, Green, Blue) vectors and $y_{1}=\left[\begin{array}{lll}0 & 1 & 0\end{array}\right]$, $y_{2}=\left[\begin{array}{lll}1 & 10\end{array}\right]$ and $y_{3}=\left[\begin{array}{lll}1 & 0 & 0\end{array}\right]$ be the vectors respectively associated with the terms Good, Medium and Bad. They respectively represent the colour Green, Yellow and Red.

Applying the previous concepts to the performance measure $p(t)$ leads to a normalised RGB vector $y$ defined as follows:

$$
\begin{gathered}
y=\delta_{2}\left(\varphi_{2}(p(t))\right)=\delta_{2}(D(p(t)))= \\
{[010] \cdot \mu_{D(p(t))}(\text { Good })+} \\
{[110] . \mu_{D(p(t))}(\text { Medium })+} \\
{[100] . \mu_{D(p(t))}(\text { Bad })}
\end{gathered}
$$

Let us illustrate the result for month 3, i.e. when the performance measure is equal to $-3.7 \%$. It was shown that the fuzzy description is:
$D(-3.7)=0.65 /$ Good $+0.35 /$ Medium $+0 /$ Bad.

It leads to the following normalised RGB vector:

$y=\left[\begin{array}{lll}0 & 1 & 0\end{array}\right] .0 .65+\left[\begin{array}{lll}1 & 1 & 0\end{array}\right] .0 .35+\left[\begin{array}{lll}1 & 0 & 0\end{array}\right] .0=\left[\begin{array}{lll}0.35 & 1 & 0\end{array}\right]$,

which corresponds to the following colour:

\subsection{Smiley-based user interface}

The idea retained for the smiley-based interface is to represent the smile by an ellipse whose semi-minor axis depends on the result of the defuzzification of the linguistic fuzzy set. Let $y_{1}=1, y_{2}=0$ and $y_{3}=-1$ be the values respectively associated with the terms Good, Medium and $B a d$. The defuzzification will lead to a number $y$ in the interval $[-1,1]$ where -1 should produce an unhappy face while the value 1 produces a happy face.

The defuzzification for the third month gives $y=0.65$ and produces the smiley given in Fig. 5.

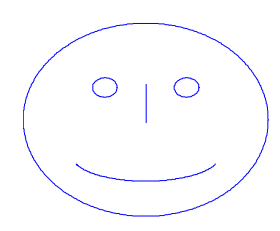

Fig. 5: The performance smiley evaluation of the third month.

\subsection{Case study performance evaluation.}

The proposed model was applied to the third month (March) of the company action plan concerning the OTD. Table 3 gathers the OTD performance which can be directly interpreted by the plant manager and the smileys respectively associated with the DPQ, QR, EPED performances.

Table 3: March scorecard for the cylinder process

Hydraulic Cylinder On Time Delivery : 47,3\%

Difference: $-2,8 \%$

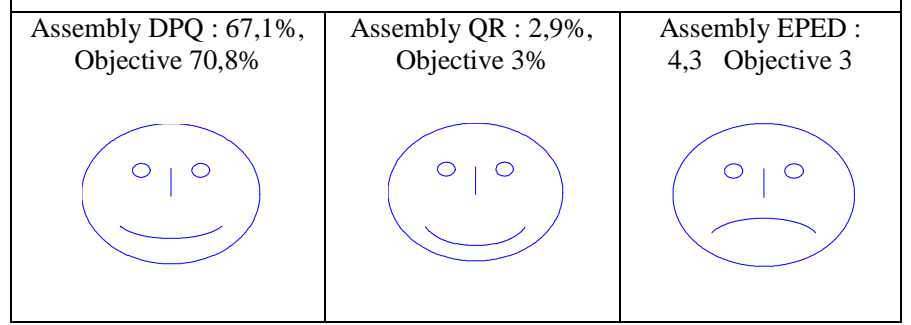

These inputs provide a scorecard whose interpretation is easier than numbers for decision-making. So the Plant Manager knows on the significant way both the tactical and the operational performance of the HC process. Note that the operational performances of the whole activities of the cylinder process must complete the scorecard. From the OTD performance $p\left(O T D, t_{3}\right)=-2,8 \%$ and the set of operational 
performance evaluations (see Table 3), namely the bad ones such as $p\left(E P E D, t_{3}\right)$, the plant manager can manage the action plan in an easier way than when he only knows the table 1 data.

\section{CONCLUSION AND FUTURE WORKS}

The performance evaluation can be an important aid for company's decision maker in the industrial improvement context. Beyond a direct measure or the computation of a comparison between measure and the fixed objective it is possible to give meaningful pieces of information according to the potential decision of the decision-maker. In this context, a fuzzification/defuzzication process has been proposed to provide symbolic information thanks to colours or smileys. The resulting symbolic information allows the decision-maker to easily make the right decision when only the measure or comparison requires analysis of their value.

Applying this proposal to a more complex real case study is the first perspective of this work. Indeed identifying the set of potential decisions and searching for the best way to represent the performance in a given industrial context could represent a real advance in the industrial improvement control. From a more academic point of view, previous works have dealt with the aggregated performance computation from elementary ones. It seems to be interesting to combine, in the aggregation process, the low level information provided by the elementary performance measures to higher level ones provided as symbolic information. Finally, the temporal aspect of the decision process should be analysed in more detail in an industrial improvement approach.

\section{REFERENCES}

[Belton and Stewart (2002)] Belton, V. and Stewart, T. J. (2002), Multiple criteria decision analysis - an integrated approach, Kluwer Academic Publishers.

[Berrah et al. (2004)] Berrah, L., Mauris, G. and Vernadat, F. (2004), 'Information aggregation in industrial performance measurement: rationales, issues and definitions', International Journal of Production Research 42(20), 4271-4293.

[Berrah et al. (1998)] Berrah, L., Mauris, G., Foulloy, L. and Haurat, A. (1998), Fuzzy performance indicators for manufacturing processes, in L. Reznik, V. Dimitrov and J. Kaprzyk, eds, 'Fuzzy System Design: Social and Engineering Applications', Vol. 17, Physica-Verlag, pp. 225-248.

[Berrah et al. (2000)] Berrah, L., Mauris, G., Haurat, A. and Foulloy, L. (2000), 'Global vision and performance indicators for an industrial improvement approach', Computers in Industry 43(3), 211-225.

[Bourguignon et al. (2004)] Bourguignon,

A., Malleret, V. and Nørreklit, H. (2004), 'The american balanced scorecard versus the french tableau de bord: the ideological dimension', Management Accounting Research 15(2), 107-134.

[Clivillé et al. (2007)] Clivillé, V., Berrah, L. and Mauris, G. (2007), 'Quantitative expression and aggregation of performance measurements based on the
MACBETH multi-criteria method', International Journal of Production Economics 105(1), 171-189.

[Epstein and Mansoni (1998)] Epstein, M. and Mansoni, J. (1998), 'Implementing corporate strategy: From tableaux de bord to balanced scorecards', European Management Journal 16(1), 190-203.

[Fortuin (1988)] Fortuin, L. (1988), 'Performance indicators, why, where and how?', European Journal of Operational Research 34, 1-9.

[Foulloy and Galichet (1995)] Foulloy, L. and Galichet, S. (1995), Typology of fuzzy controllers, in H. Nguyen, M. Sugeno, R. Tong and R. Yager, eds, 'Theoretical Aspects of Fuzzy Control', John Wiley \& Sons, pp. 6590.

[Gomez et al. (2001)] Gomez, T., Gonzalez, M. and Luque, M. (2001), 'Multiple objectives decompositioncoordination methods for hierarchical organizations', European Journal of Operational Research 133, 323341.

[Kaplan and Norton (1992)] Kaplan, R. and Norton, D. (1992), The Balanced Scorecard: Measures that drive performances, Harvard Business Review.

[Kaplan and Norton (1996)] Kaplan, R. and Norton, D. (1996), The Balanced Scorecard: Translating Strategy into Action, Harvard Business School Press, Boston.

[Lebas (1995)] Lebas, M. (1995), 'Performance measurement and performance management', International Journal of Production Economics 1-3, 2335.

[Nair (2006)] Nair, A. (2006), 'Meta-analysis of the relationship between quality management practices and firm performance-implications for quality management theory development', Journal of Operations Management 24(6), 948-975.

[Neely et al. (1995)] Neely, A., Gregory, M. and Platts, K. (1995), 'Performance measurement system design: a literature review and research agenda', International Journal of Operations and Production Management 48(4), 80-116.

[Nudurupati et al. (2011)] Nudurupati, S. S., Bititci, U. S., Kumar, V. and Chan, F. T. S. (2011), 'State of the art literature review on performance measurement', Computers \& Industrial Engineering 60(2), 279-290.

[Ruspini (1969)] Ruspini, E. (1969), 'A new approach to clustering', Information and Control 15(1), 22-32.

[Simon (1977)] Simon, H. (1977), The new science of management decision, Prentice-Hall.

[Vallespir et al. (1993)] Vallespir, B., Merle, C. and Doumeingts, G. (1993), 'Gim: A technico-economic methodology to design manufacturing systems', Control Engineering Practice 1(6), 1031-1038.

[Zadeh (1965)] Zadeh, L. (1965), 'Fuzzy sets', Information and Control 8, 38-353.

[Zadeh (1971)] Zadeh, L. (1971), 'Quantitative fuzzy semantics', Information Sciences 3, 159-176. 